\title{
Effects of radial electric field on suppression of electron-temperature- gradient mode through multiscale nonlinear interactions
}

\author{
Chanho Moon ${ }^{1,2, a)}$, Toshiro Kaneko ${ }^{1}$, Kimitaka Itoh $^{2}$, Katsumi Ida $^{2}$, Tatsuya Kobayashi ${ }^{2}$, \\ Shigeru Inagaki ${ }^{3}$, Sanae-I. Itoh $^{3} \&$ Rikizo Hatakeyama ${ }^{1}$ \\ ${ }^{1}$ Department of Electronic Engineering, Tohoku University, Sendai 980-8579, Japan \\ ${ }^{2}$ National Institute for Fusion Science, Toki, Gifu 509-5292, Japan \\ ${ }^{3}$ Research Institute for Applied Mechanics, Kyushu University, Kasuga 816-8580, Japan
}

\begin{abstract}
Turbulence in fluids and plasmas is ubiquitous in nature and laboratory. Contrary to the importance of the 'scale-free' nature of cascade in neutral fluid turbulence, the turbulence in plasma is characterised by dynamics of distinct length scales. The cross-scale interactions can be highly non-symmetric so as to generate the plasma turbulence structures. Here we report that the system of hyper-fine electron-temperature-gradient (ETG) fluctuations and microscopic drift-wave (DW) fluctuations is strongly influenced by the sign of the gradient of the radial electric field through multiscale nonlinear interactions. The selective suppression effects by radial electric field inhomogeneity on DW mode induce a new route to modify ETG mode. This suppression mechanism shows disparity with respect to the sign of the radial electric field inhomogeneity, which can be driven by turbulence, so that it could be a new source for symmetry breaking in the turbulence structure formation in plasmas.
\end{abstract}

a) Present address: Max-Planck-Institut für Plasmaphysik, EURATOM Association, 85740 Garching, Germany; electric mail:chanho.moon@ipp.mpg.de 
Magnetized plasmas with a significant temperature gradient across the magnetic field are commonly observed in laboratory and nature, and this temperature gradient drives the turbulent fluctuations. In particular, anomalous electron heat transports induced by the electron-temperature-gradient (ETG) mode in magnetized plasma [1-3] have attracted attention. Contrary to its possible importance in the turbulence-driven transport, the nonlinear evolution of ETG modes is understood less well in comparison with the rapid progress in understanding drift-wave (DW) [4]. The characteristic features of nonlinear interactions in ETG fluctuations are (i) predicted not to be effectively suppressed by radial electric field $\left(E_{\mathrm{r}}\right)$ driven shear $\left(E_{\mathrm{r}}^{\prime}\right)$, i.e., $E \times B$ velocity shear ( $B$ is the magnetic field), because the ETG mode is characterised by the smaller spatial scales and larger growth rate [5,6]. And (ii) the radiallyextended fluctuations have been predicted to emerge from hyper-fine scale ETG mode $[7,8]$. As a consequence, the study of ETG mode requires the multiscale nonlinear interactions, such as ETG-DW, ETG-DW-global $E_{\mathrm{r}}$ and others. Furthermore, recent experimental results have shown that the ETG mode has a nonlinear coupling with the low-frequency fluctuations, such as the DW mode [9] and the ion acoustics mode [10]. In particular, we previously report that the DW mode is originally stable in the equilibrium plasma state, and is enhanced via nonlinear coupling with the ETG mode when the amplitude of the ETG mode exceeds a certain threshold [9]. Therefore, it is necessary to consider the multiscale fluctuations and nonlinear interactions $[11,12]$ for clearly understanding the suppression mechanism of the ETG mode. In addition, empirical observations indicate that the effect of $E_{\mathrm{r}}$ driven shear on fluctuations relies on the sign of the $E_{\mathrm{r}}^{\prime}[13,14]$, which is also one of the most urgent issues for understanding the suppression mechanism of plasma fluctuations. Specifically, it is predicted that the radial electric field inhomogeneity is the most important factor to suppress the plasma fluctuations [23].

Recently, Smith et al. reported that the sufficiently large velocity-shear of the mean $E \times B$ 
drift could be an effective suppression mechanism for the ETG mode [15]. However, the experimental investigation of the nonlinear interaction of ETG mode and mean radial electric field demands further detailed studies. First, not only the isolated interaction between the ETG mode and the $E_{\mathrm{r}}$, but also the chained-interactions among the ETG mode, fluctuations at ion gyroradius, and the $E_{\mathrm{r}}$ must be investigated. Second, the influence of the shear and the curvature of the electric field must be separately studied. These demand the accessibility of fluctuation diagnostics over the plasma column and detailed controllability of $E_{\mathrm{r}}$. For this purpose, the use of precise measurements on the ETG mode in a linear laboratory device is advantageous in advancing the understanding of the ETG mode and the structure formation [16]. This merit is unique, even though the ETG mode in the slab cylindrical plasma is not identical to the toroidal ETG mode. Here we demonstrate a new mechanism for the ETG mode suppression through the multiscale nonlinear coupling processes, which in turn affects the ETG mode amplitude. This new mechanism will help to understand the underlying fundamental nonlinear mechanism of modern plasma physics.

Experiments were performed on the $Q_{\mathrm{T}}-$ Upgrade machine at Tohoku University. Figure 1 shows a schematic of the experimental apparatus. One section is the source region where an electron cyclotron resonance $(\mathrm{ECR})$ plasma with relatively high electron temperature $\left(T_{\mathrm{e}} \simeq 3\right.$ $\mathrm{eV}$ ) is generated, and the other section is the experimental region where low-temperature thermionic electrons $\left(T_{\mathrm{e}}=0.2 \mathrm{eV}\right)$ are superimposed on the high-temperature electrons of the ECR plasma. The end of the experimental region is used as an electron emitter and is heated by applying DC power $P_{\mathrm{HP}}=3 \mathrm{~kW}$ in order to generate low-temperature thermionic electrons. The ETG can be controlled using the two differently shaped stainless-steel (SUS) mesh grids, which are located at $z \simeq-40 \mathrm{~cm}$ and separate the source region from the experimental region. The high-temperature electrons of the ECR plasma pass through the grids with negative biases, which are used to adjust the radial density profile of the high-temperature 
electrons in the experimental region. This novel plasma source affords a method suitable for forming and controlling the ETG [16]. Hereinafter, the voltages applied to the mesh grids are defined as $V_{\mathrm{g} 1}$ (grid 1) and $V_{\mathrm{g} 2}$ (grid 2). Furthermore, the hot plate as the electron emitter is concentrically segmented into three sections, the outermost section of which is always grounded in this experiment. A radial profile of plasma space potential, namely, the $E_{\mathrm{r}}$ can be controlled independently of the ETG by changing bias voltages of the electron emitter, and the bias voltages applied to the central $\left(V_{\text {ee1 }}\right)$ and peripheral $\left(V_{\mathrm{ee} 2}\right)$ sections are defined as $V_{\text {ee1 }}$ and $V_{\mathrm{ee} 2}$. In order to measure the precise profiles of the ETG modes, Langmuir probes are made with probe tip diameter $\sim 0.125 \mathrm{~mm}$ and length $\sim 1.0 \mathrm{~mm}$, which is small enough to obtain the small spatial scale of the ETG mode.

Figure 2 presents radial profiles of electron temperature $\left(T_{\mathrm{e}}\right)$ and space potential $\left(\phi_{\mathrm{s}}\right)$ for (a) $V_{\text {ee1 }}=-3 \mathrm{~V}$ (hill type), (b) $V_{\text {eel }}=-4 \mathrm{~V}$ (flat type) and (c) $V_{\text {eel }}=-5 \mathrm{~V}$ (well type) for $\nabla T_{\mathrm{e}} / T_{\mathrm{e} 0} \simeq 0.8 \mathrm{~cm}^{-1}, P_{\mu}=20 \mathrm{~W}$ and magnetic fields $B=(0.21-0.23) \mathrm{T}$. The $\nabla T_{\mathrm{e}} / T_{\mathrm{e} 0}$ is obtained as the local electron temperature gradient in the region of $\left|r_{\mathrm{m}}\right|=0.5 \mathrm{~cm} \sim 2.0 \mathrm{~cm}$ calculated from the radial profiles of the electron temperature, where the $T_{\mathrm{e} 0}$ is the core electron temperature. Here, the axial center of the machine is defined as $z=0 \mathrm{~cm}$ and the $r_{\mathrm{m}}$ indicates the measurement radial position of probes in the experimental region. By decreasing the applied bias voltage $V_{\text {eel } 1}$, the $\phi_{\mathrm{s}}$ only in the central region $\left(\left|r_{\mathrm{m}}\right|<1.0 \mathrm{~cm}\right)$ is changed definitely, while the $\phi_{\mathrm{s}}$ in the peripheral region $\left(\left|r_{\mathrm{m}}\right|>1.0 \mathrm{~cm}\right)$ is almost unchanged. Consequently, the $E_{\mathrm{r}}$ can be independently controlled without changing the ETG formation, because the electron temperature is not affected by the change of $V_{\mathrm{ee} 1}$. Furthermore, a relatively gentle density gradient of $L_{\mathrm{n}} \simeq 4 \mathrm{~cm}\left(>L_{\mathrm{Te}} \sim 1.2 \mathrm{~cm}\right)$ persists even when the $\phi_{\mathrm{s}}$ profiles are changed, where $L_{\mathrm{n}}$ and $L_{\mathrm{Te}}$ are defined as the density and temperature gradient scale lengths, respectively. The other parameters are the electron collision frequency $v_{e i} \sim 4.3 \mathrm{kHz}$, the drift scale $\rho_{s}\left(c_{S} /\right.$ 
$\left.\Omega_{i}\right) \sim 3.4 \mathrm{~cm}$, and $\eta_{\mathrm{e}} \sim 3.3$. This is very useful for investigation of the effects of $E_{\mathrm{r}}$ on the suppression of the ETG mode with great precision. The average $E_{\mathrm{r}}$ is calculated by dividing plasma space potential difference $\left(\Delta \phi_{\mathrm{s}}\right)$ between the central $\left(r_{\mathrm{m}} \simeq-0.6 \mathrm{~cm}\right)$ and peripheral $\left(r_{\mathrm{m}}\right.$ $\simeq-1.6 \mathrm{~cm}$ ) regions by $\Delta r=1 \mathrm{~cm}$ at $z=50 \mathrm{~cm}$. Since the $\phi_{\mathrm{s}}$ profiles are dominantly changed in the region $-1.6 \mathrm{~cm}<r_{\mathrm{m}}<-0.6 \mathrm{~cm}$, the $E_{\mathrm{r}}$ value can be determined through the average $E_{\mathrm{r}}$ calculation $(=-\partial \phi(r) / \partial r)$ in the experimental conditions. The $E_{\mathrm{r}}$ values in Figs. 2(a), (b) and (c) become approximately $0.8 \mathrm{~V} / \mathrm{cm},-0.2 \mathrm{~V} / \mathrm{cm}$ and $-1.1 \mathrm{~V} / \mathrm{cm}$, respectively.

Figure 3 shows the electron saturation current fluctuations $\tilde{I}_{e s}$ with (a) high and (b) low frequencies as a function of $E_{\mathrm{r}}$ for $\nabla T_{\mathrm{e}} / T_{\mathrm{e} 0} \simeq 0.8 \mathrm{~cm}^{-1}$ at $r_{\mathrm{m}}=-0.9 \mathrm{~cm}$. The high-frequency fluctuation, namely, the ETG mode, varies sensitively owing to the azimuthal Doppler shift by changing the $E_{\mathrm{r}}$, as shown in Fig. 3(a). The $E \times \mathrm{B}$ drift velocity caused by the radial profile of plasma potential corresponds to the electron diamagnetic direction when the $E_{\mathrm{r}}$ becomes negative value [see Fig. 2(c)]. In the case of $E_{\mathrm{r}} \sim-0.6 \mathrm{~V} / \mathrm{cm}$, the Doppler shift frequency of the ETG mode becomes approximately $1.4 \mathrm{kHz}$ which consistent with the increase in the peak frequencies of ETG modes with $E_{\mathrm{r}}$. Based on the results, it is confirmed that the ETG mode propagates in the same direction as the electron diamagnetic direction. Furthermore, the ETG mode amplitude is decreased in the slightly negative $E_{\mathrm{r}}$, while the ETG mode amplitude is increased in the slightly positive $E_{\mathrm{r}}$. On the other hand, the amplitude of the low-frequency fluctuation with $f \simeq 7 \mathrm{kHz}$, namely the DW mode amplitude, is decreased in the slightly positive $E_{\mathrm{r}}$, while it is increased in the slightly negative $E_{\mathrm{r}}$, as shown in Fig. 3(b). Moreover, the Doppler shift frequency of the DW mode becomes approximately $0.23 \mathrm{kHz}$ in the case of $E_{\mathrm{r}} \sim-0.6 \mathrm{~V} / \mathrm{cm}$, which is consistent with the small change in the peak frequencies of DW modes (electron diamagnetic direction). Therefore, it is considered likely that the Doppler shift are negligible for the DW mode growth rate $(0.23 \mathrm{kHz}<<7 \mathrm{kHz})$. Additionally, the 
Doppler shear is approximately three times larger than the Doppler shift value, so that would be also negligible.

Furthermore, it was confirmed that the azimuthal wave number of the ETG mode $\left(k_{\theta} \simeq\right.$ $\left.30 \mathrm{~cm}^{-1}\right)$ was much larger than the wave number of the DW mode $\left(k_{\theta} \simeq 5 \mathrm{~cm}^{-1}\right)$ through measurement by twin probes with a distance of $3 \mathrm{~mm}$. The frequency of the ETG mode at the wave number $k_{\theta} \simeq 30 \mathrm{~cm}^{-1}$ corresponds to the theoretically estimated slab ETG mode dispersion frequency [17], and the radial profiles of the normalized amplitudes of the ETG mode and the DW mode are almost constant when the $E_{\mathrm{r}}$ is changed

Figure 4 presents the normalized amplitudes $\tilde{I}_{e s} / \bar{I}_{e s}$ of fluctuations with high and low frequencies $\left(\bar{I}_{e s}\right.$ : the mean value of the electron saturation current $\left.I_{e s}\right)$ for (a) $\nabla T_{\mathrm{e}} / T_{\mathrm{e} 0} \simeq 0.34$ $\mathrm{cm}^{-1}$ (in the case of the small ETG) and (b) $\nabla T_{\mathrm{e}} / T_{\mathrm{e} 0} \simeq 0.8 \mathrm{~cm}^{-1}$ (in the case of the large ETG) as a function of $E_{\mathrm{r}}$ at $r_{\mathrm{m}}=-0.9 \mathrm{~cm}$. The value of $\tilde{I}_{e s} / \bar{I}_{e s}$ is calculated from the peak amplitude of the spectrum with a frequency of $\sim 0.4 \mathrm{MHz}$ (for ETG mode), and with a frequency of $\sim 7 \mathrm{kHz}$ (for DW mode). In the case of the small ETG, the ETG mode is symmetrically suppressed by the $E \times B$ drift velocity shear owing to the sufficiently large $E_{\mathrm{r}}$, which is consistent with the theoretical estimation by Gao et al. $[5,6]$. Specifically, the ETG mode cannot be stabilized until the $E \times B$ drift velocity shear becomes two times larger than $v_{\mathrm{i}} / L_{\mathrm{n}} \sim 3 \times 10^{4} \mathrm{~s}^{-1}$, where $v_{\mathrm{i}}$ is ion thermal speed. Therefore, the sufficiently large $\left|E_{\mathrm{r}}\right|>\sim 0.7$ V/cm driven large $E \times B$ drift velocity shear $\left(\sim 7 \times 10^{4} \mathrm{~s}^{-1}\right)$ is in agreement with the theoretical estimation for the threshold values of the ETG mode suppression. In addition, the DW mode decreases with an increase in the $E_{\mathrm{r}}$ regardless of its sign, which is also consistent with the numerical calculations on the effect of $E_{\mathrm{r}}$ on the DW mode $[18,19]$. Therefore, for the small ETG, i.e., $\nabla T_{\mathrm{e}} / T_{\mathrm{e} 0} \simeq 0.34 \mathrm{~cm}^{-1}$, the stabilized characteristics of the ETG mode and the DW mode on the effect of $E_{\mathrm{r}}$ are in agreement with the conventional theoretical results. 
On the other hand, in the case of the larger ETG, i.e., $\nabla T_{\mathrm{e}} / T_{\mathrm{e} 0} \simeq 0.8 \mathrm{~cm}^{-1}$, the stabilized characteristics of the ETG mode and the DW mode on the effect of $E_{\mathrm{r}}$ are significantly different from those in the case of the small ETG [Fig. 4(b)]. It is observed that the $\tilde{I}_{e s} / \bar{I}_{e s}$ of the ETG mode is increased in the slightly positive $E_{\mathrm{r}}$, while it is decreased in the negative $E_{\mathrm{r}}$. In contrast, the $\tilde{I}_{e s} / \bar{I}_{e s}$ of the DW mode is decreased in the slightly positive $E_{\mathrm{r}}$, while it is increased in the negative $E_{\mathrm{r}}$. It is to be noted that the dependence of the ETG mode on $E_{\mathrm{r}}$ is definitely different from the case of the DW mode. This difference is considered to be caused by the nonlinear interaction between the ETG mode and the DW mode.

To evaluate the degree of the nonlinear coupling between the ETG and the DW modes we compute the auto-bicoherence (normalized bispectrum), which is an indicator of the strength of nonlinear three-wave couplings [20]. Figure 5 gives the auto-bicoherence of the electron saturation current fluctuations $\left(\tilde{I}_{e s}\right)$ for bicoherence planes of (a) $E_{\mathrm{r}} \simeq-1.0 \mathrm{~V} / \mathrm{cm}$ and (b) $E_{\mathrm{r}} \simeq+1.0 \mathrm{~V} / \mathrm{cm}$ for $\nabla T_{\mathrm{e}} / T_{\mathrm{e} 0} \simeq 0.8 \mathrm{~cm}^{-1}$ at $r_{\mathrm{m}}=-0.9 \mathrm{~cm}$. The frequency resolution of the bicoherence is $\Delta f=1 \mathrm{kHz}$. For $E_{\mathrm{r}} \simeq-1.0 \mathrm{~V} / \mathrm{cm}$, it can be seen that the significant nonlinear coupling between fluctuations pairs with $f \simeq 0.4 \sim 0.8 \mathrm{MHz}$ and $7 \mathrm{kHz}$ is observed in a broad frequency region, where the colour gradient on the right indicates the level of bicoherence. This corresponds to the fact that a nonlinear coupling takes place between the broadband ETG mode and the DW mode when the negative $E_{\mathrm{r}}$ is formed. On the other hand, for $E_{\mathrm{r}} \simeq$ $+1.0 \mathrm{~V} / \mathrm{cm}$, the significant nonlinear coupling between the fluctuations with $f \simeq 0.4 \mathrm{MHz}$ and $7 \mathrm{kHz}$ is observed in a narrow frequency region. Therefore, the relative strength of nonlinear coupling can be determined by the integrated bicoherence in the range of the ETG and the DW modes frequencies.

Figure 6(a) shows the $E_{\mathrm{r}}$ dependence of the integrated bicoherence in the range of $f_{1} \simeq$ $0.4 \sim 0.8 \mathrm{MHz}$, where $f_{2}$ is $6 \sim 8 \mathrm{kHz}$ (DW mode) in order to quantitatively investigate the 
nonlinear coupling with respect to the ETG mode. The integrated bicoherence is defined as $b^{2}=\iint_{D} \hat{b}^{2}\left(f_{1}, f_{2}\right) d f_{1} d f_{2}\left(\mathrm{D}: f_{1} \simeq 0.4 \sim 0.8 \mathrm{MHz}, f_{2} \simeq 6 \sim 8 \mathrm{kHz}\right)$, which provides an indication of the relative amount of the nonlinear coupling between the ETG and the DW modes. The value of integrated bicoherence between the two modes is very low over the whole $E_{\mathrm{r}}$ region in the case of $\nabla T_{\mathrm{e}} / T_{\mathrm{e} 0} \simeq 0.34 \mathrm{~cm}^{-1}$. In the case of $\nabla T_{\mathrm{e}} / T_{\mathrm{e} 0} \simeq 0.8 \mathrm{~cm}^{-1}$, on the other hand, the bicoherence suddenly increases when the $E_{\mathrm{r}}$ becomes negative value. Therefore, it is concluded that when the $E_{\mathrm{r}}$ becomes slightly positive, the $\tilde{I}_{e s} / \bar{I}_{e s}$ of the DW mode is suppressed owing to the effect of $E_{\mathrm{r}}$ (the $E \times B$ drift velocity shear), corresponding to the decrease of the nonlinear coupling between the ETG and the DW modes. Consequently, the $\tilde{I}_{e s} / \bar{I}_{e s}$ of the ETG mode is increased. However, interestingly, when the $E_{\mathrm{r}}$ becomes slightly negative, the $\tilde{I}_{e s} / \bar{I}_{e s}$ of the ETG mode is decreased and the DW mode is increased. In Fig. $6(\mathrm{~b})$, the normalized $E \times B$ shearing rate $\omega_{\mathrm{E} 1 * \tau_{\mathrm{cDW}}}$ is estimated using the experimental results, where $\omega_{\mathrm{E} 1}=\partial v_{\mathrm{E}} / \partial r\left(v_{\mathrm{E}}=E_{\mathrm{r}} / \mathrm{B}_{0}\right.$ is the $E \times B$ drift velocity $)$ is $E \times B$ drift velocity shear, and $\tau_{\mathrm{cDW}}$ is the decorrelation time of the DW mode. The comparison of the large and the small ETG shows that the normalized $E \times B$ shearing rate is decreased a little when the $E_{\mathrm{r}}$ becomes slightly negative in the case of $\nabla T_{\mathrm{e}} / T_{\mathrm{e} 0} \simeq 0.8 \mathrm{~cm}^{-1}$. However, the value of the $E \times B$ drift velocity shear $\omega_{\mathrm{E} 1}$ becomes comparable to the decorrelation time of the DW mode $\tau_{\mathrm{cDW}}$ for $E_{\mathrm{r}}<\sim-0.5 \mathrm{~V} / \mathrm{cm}$, which is insufficient to explain the $\tilde{I}_{e s} / \bar{I}_{e s}$ of the DW mode increasing. Recently, empirical evidence suggest that the mean flow $\alpha\left(-E_{\mathrm{r}} E_{\mathrm{r}}\right)$ affects the plasma fluctuations [13]. Thus we derive the radial profile of the radial electric field $E_{\mathrm{r}}$, the normalized $E \times B$ shearing rate $\omega_{\mathrm{E} 1 * \tau_{\mathrm{cDW}}}$ and the mean flow $-E_{\mathrm{r}} E_{\mathrm{r}}{ }_{\mathrm{r}}$ from the radial profile of $\phi_{\mathrm{s}}$ in the region of $\left|r_{\mathrm{m}}\right|=0.6 \mathrm{~cm} \sim 1.6 \mathrm{~cm}$, as shown in Fig. 7. The $-E_{\mathrm{r}} E_{\mathrm{r}}^{\prime \prime}$ values become substantially negative when $E_{\mathrm{r}}$ is inner direction, as shown in Fig. 7(b), which is possibly causing the plasma fluctuation. Therefore, the nonlinear coupling between the ETG and the 
DW modes is increased, and this nonlinear coupling then enhances the energy transfer from the ETG mode to the DW mode, causing the effective suppression of the former even in the small negative $E_{\mathrm{r}}$. These experimental results are consistent with the theoretical expectation of the impact of $E_{\mathrm{r}}$ on the multiscale nonlinear interaction [7].

We showed that the $E_{\mathrm{r}}$ could be controlled independently of the ETG by changing the bias voltages of the electron emitter. It is found that a sufficiently large $E_{\mathrm{r}}$ can suppress the ETG mode regardless of its signs in the case of the small ETG. However, for the large ETG case, even when $E_{\mathrm{r}}$ becomes small, the ETG mode amplitude is decreased in the slightly negative $E_{\mathrm{r}}$, while the ETG mode amplitude remains large in the positive $E_{\mathrm{r}}$. In this case, the DW mode that is enhanced by the nonlinear coupling with the ETG mode has the opposite tendency of dependence on $E_{\mathrm{r}}$, namely, the DW mode amplitude increases and decreases in the cases of the negative and the positive $E_{\mathrm{r}}$, respectively. Since a bicoherence between the high-frequency ETG and low-frequency DW modes is observed to increase with the slightly negative $E_{\mathrm{r}}$ in the case of the large ETG, the ETG mode amplitude is decreased by the energy transfer from the ETG mode to the DW mode through the multiscale nonlinear coupling. It can be shown for the first time that the $E_{\mathrm{r}}$ affects turbulent fluctuations through not only the direct suppression but also the nonlinear interaction of the ETG and the DW modes, which consequently caused the new suppression mechanism of the ETG mode. This suppression mechanism shows disparity with respect to the sign of the radial electric field inhomogeneity, which can be driven by turbulence, so that it could be a new source for symmetry breaking in the turbulence structure formation in plasmas. The results contain the significant implication that the radial electric field inhomogeneity is the most important factor in the suppression mechanism for the plasma fluctuations.

\section{Acknowledgements}


This work was supported by the Grant-in-Aid for JSPS Fellows (23-1638) and a Grant-in-Aid for Scientific Research from the Ministry of Education, Culture, Sports, Science and Technology, Japan. This work is partly supported by the Grant-in-Aid for Scientific Research of JSPS, Japan (Nos. 15H02155 and 23244113), by the collaboration programs of the RIAM of Kyushu University and of NIFS (NIFS13KOCT001), and by the Asada Science Foundation.

\section{References}

[1] Mattoo S K et al 2012 Phys. Rev. Lett. 108, 25507

[2] Sokolov V and Sen A K 2011 Phys. Rev. Lett. 107, 155001

[3] Mazzucato E et al 2008 Phys. Rev. Lett. 101, 075001

[4] Horton W 1999 Rev. Mod. Phys. 71, 735-778

[5] Gao Z et al 2003 Phys. Plasmas 10, 2831

[6] Gao Z et al 2004 Phys. Plasmas 11, 3053

[7] Itoh S-I and Itoh K 2001 Plasma Phys. Control. Fusion 43, 1055

[8] Holland C and Diamond P H 2005 Phys. Lett. A 344, 369-382

[9] Moon C, Toshiro K and Hatakeyama R 2013 Phys. Rev. Lett. 111, 115001

[10] Sokolov V and Sen A K 2014 Phys. Rev. Lett. 113, 095001

[11] Porkolab M and Chang R P H 1978 Rev. Mod. Phys. 50, 745-795

[12] Robinson P A 1997 Rev. Mod. Phys. 69, 507-573

[13] Tokuzawa T et al 2014 Phys. Plasmas 21, 055904

[14] Moyer R A et al 1995 Phys. Plasmas 2, 2397

[15] Smith D R et al 2009 Phys. Rev. Lett. 102, 225005

[16] Moon C et al 2010 Rev. Sci. Instrum. 81, 053506

[17] Lee Y C et al 1987 Phys. Fluids 30, 1331

[18] Chaudhry M B et al 1988 J. Phys. Soc. Jpn. 57, 3043

[19] Sanuki H 1984 Phys. Fluids 27, 2500

[20] Kim Y C and Powers E J 1979 IEEE Trans. Plasma Sci. PS-7, 120

[21] Diamond P H et al 2005 Phys. Control. Fusion 47, R35

[22] Itoh K et al 2015 Plasma Phys. Control. Fusion 57, 075008

[23] Itoh K and Itoh S-I 2016 Plasma Phys. Control. Fusion 58, 045017 


\section{Figures legends}

Figure 1. Schematic diagram of the experimental apparatus. We used the Cartesian coordinate to explain the straight cylinder device, which is indicated in the center of the schematic diagram.

Figure 2. Radial profiles of electron temperature $\left(T_{\mathrm{e}}\right)$ and space potential $\left(\phi_{\mathrm{s}}\right)$ for (a) hill type $\left(E_{\mathrm{r}}>0\right)$, (b) flat type $\left(E_{\mathrm{r}} \sim 0\right)$ and (c) well type $\left(E_{\mathrm{r}}<0\right)$ for $\nabla T_{\mathrm{e}} / T_{\mathrm{e} 0} \simeq 0.8 \mathrm{~cm}^{-1}$ and $P_{\mu}=20 \mathrm{~W}$. Langmuir probes are used to measure the $T_{\mathrm{e}}$ and the $\phi_{\mathrm{s}}$ profiles, which are scrutinized based on the reproducibility of the plasma parameter profiles to select the final data set. The uncertainty of the evaluation of $E_{\mathrm{r}}$ is small and may be ignored, since the uncertainty of the $E_{\mathrm{r}}$ values mostly comes from the properties of radial profiles of $\phi_{\mathrm{s}}$.

Figure 3. Dependence of the electron saturation current fluctuations $\tilde{I}_{e s}$ on the radial electric field ( $E_{\mathrm{r}}$ ) with (a) high and (b) low frequencies for $\nabla T_{\mathrm{e}} / T_{\mathrm{e} 0} \simeq 0.8 \mathrm{~cm}^{-1}$ at $r_{\mathrm{m}}$ $=-0.9 \mathrm{~cm}$. (In both figures, zero amplitude is offset for three cases in order to highlight the difference of the three spectra amplitudes.)

Figure 4. The normalized amplitudes $\tilde{I}_{e s} / \bar{I}_{e s}$ of fluctuations with high and low frequencies of (a) $\nabla T_{\mathrm{e}} / T_{\mathrm{e} 0} \simeq 0.34 \mathrm{~cm}^{-1}$ and (b) $\nabla T_{\mathrm{e}} / T_{\mathrm{e} 0} \simeq 0.8 \mathrm{~cm}^{-1}$ as a function of the $E_{\mathrm{r}}$ at $r_{\mathrm{m}}=-0.9 \mathrm{~cm}$. Since the amplitude of $E \times B$ drift velocity shear is proportional to $E_{\mathrm{r}}$ value, the $E \times B$ drift velocity shear become approximately $+9.7 \times 10^{4} \mathrm{~s}^{-1}\left(E_{\mathrm{r}} \sim+0.8 \mathrm{~V} / \mathrm{cm}\right)$ and $-1.3 \times 10^{5} \mathrm{~s}^{-1}\left(E_{\mathrm{r}} \sim-1.1 \mathrm{~V} / \mathrm{cm}\right)$ at $r_{\mathrm{m}} \simeq-0.9 \mathrm{~cm}$ (the magnetic field, $\mathrm{B}=0.214 \mathrm{~T}$ ).

Figure 5. Auto-bicoherence of the electron saturation current fluctuations $\left(\tilde{I}_{e s}\right)$ for bicoherence planes of (a) $E_{\mathrm{r}} \simeq-1.0 \mathrm{~V} / \mathrm{cm}$ and (b) $E_{\mathrm{r}} \simeq+1.0 \mathrm{~V} / \mathrm{cm}$ for $\nabla T_{\mathrm{e}} / T_{\mathrm{e} 0} \simeq$ $0.8 \mathrm{~cm}^{-1}$ at $r_{\mathrm{m}}=-0.9 \mathrm{~cm}$.

Figure 6. (a) The integrated bicoherence between ETG and drift wave modes and (b) the

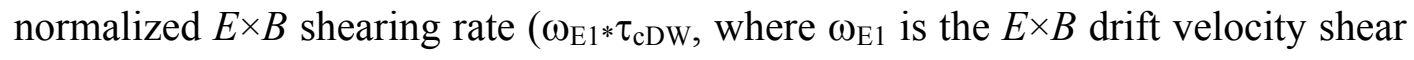
and $\tau_{\mathrm{cDW}}$ is the decorrelation time of the drift wave modes) as a function of the $E_{\mathrm{r}}$ at $r_{\mathrm{m}}=-0.9 \mathrm{~cm}$.

Figure 7. Derived radial profiles of plasma space potential $\left(\phi_{\mathrm{s}}\right)$, radial electric field $\left(E_{\mathrm{r}}\right)$, 


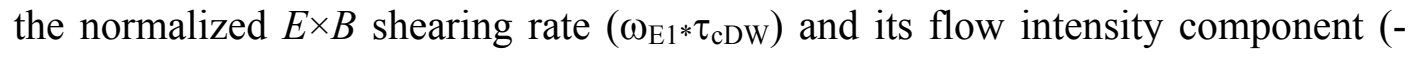
$E_{\mathrm{r}} E_{\mathrm{r}}$ ) for (a) $E_{\mathrm{r}} \simeq+0.8 \mathrm{~V} / \mathrm{cm}$ and (b) $E_{\mathrm{r}} \simeq-1.1 \mathrm{~V} / \mathrm{cm}$ computed using plasma space potential profiles (the blue marks, $-3.0 \mathrm{~cm}<r_{\mathrm{m}}<0 \mathrm{~cm}$ ) of Fig. 2(a) and Fig. 2(c). The error bars are estimated from the uncertainty of fitting parameter (the degree of a polynomial) of the $\phi_{\mathrm{s}}$ measurement profiles to the polynomial curve fitting process. We used shading in the figures to highlight where the low frequency fluctuations have a large value. 


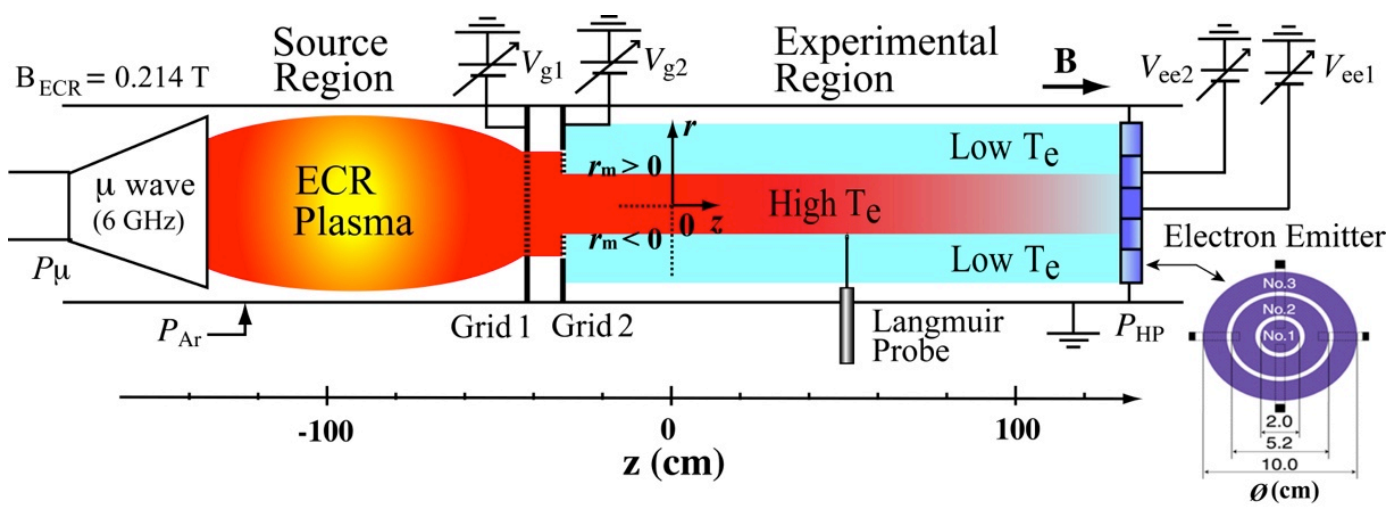

Figure 1. 

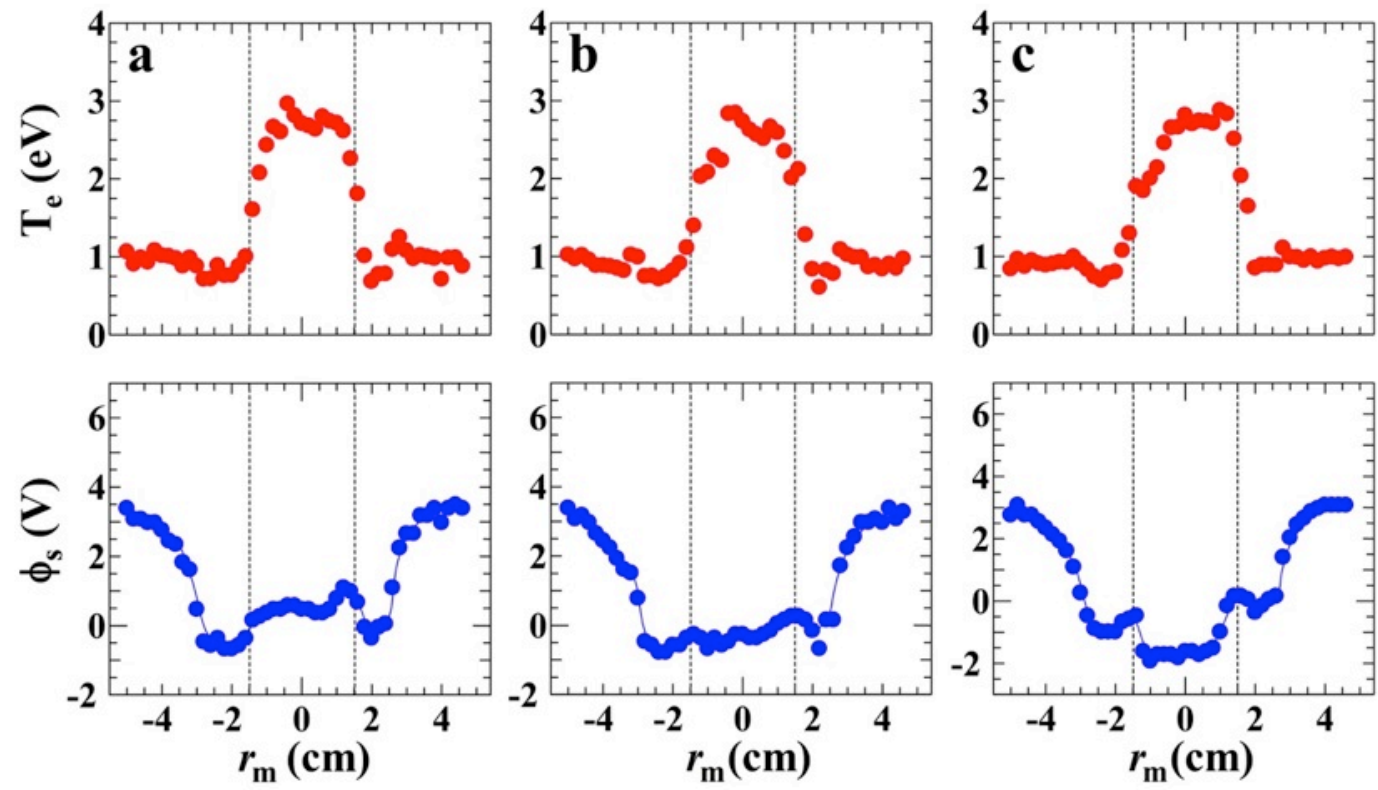

Figure 2. 

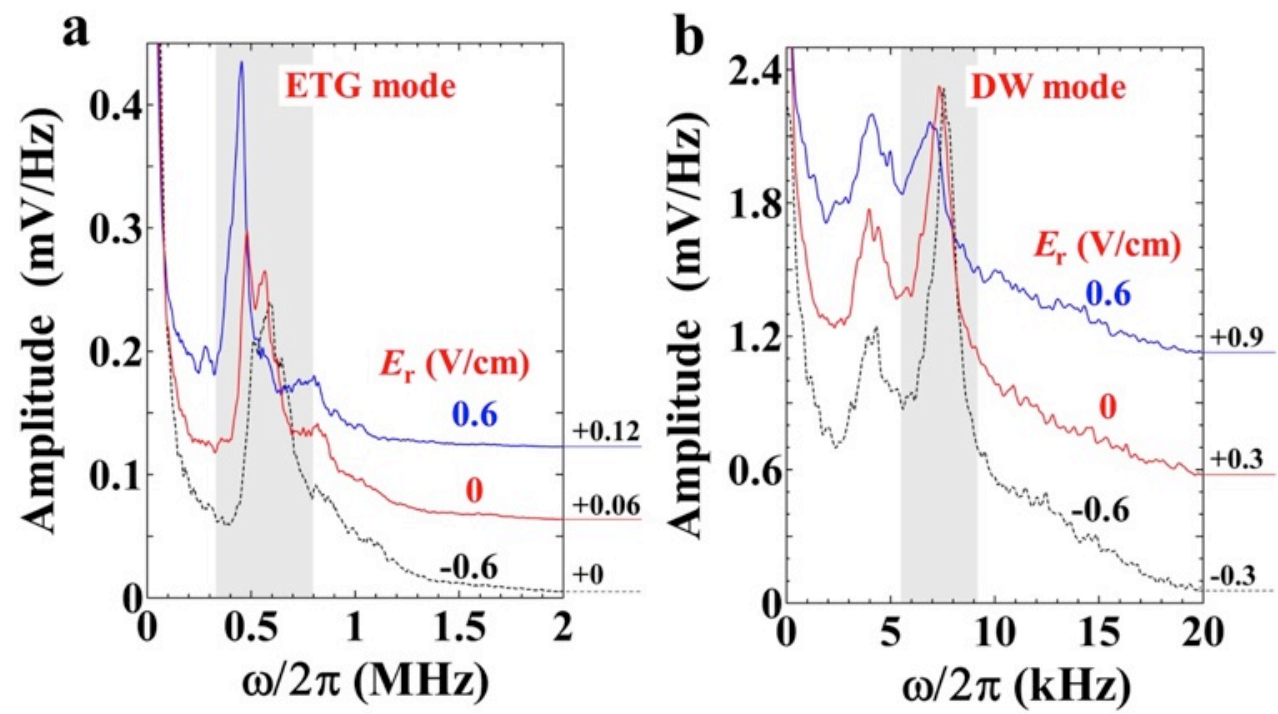

Figure 3. 


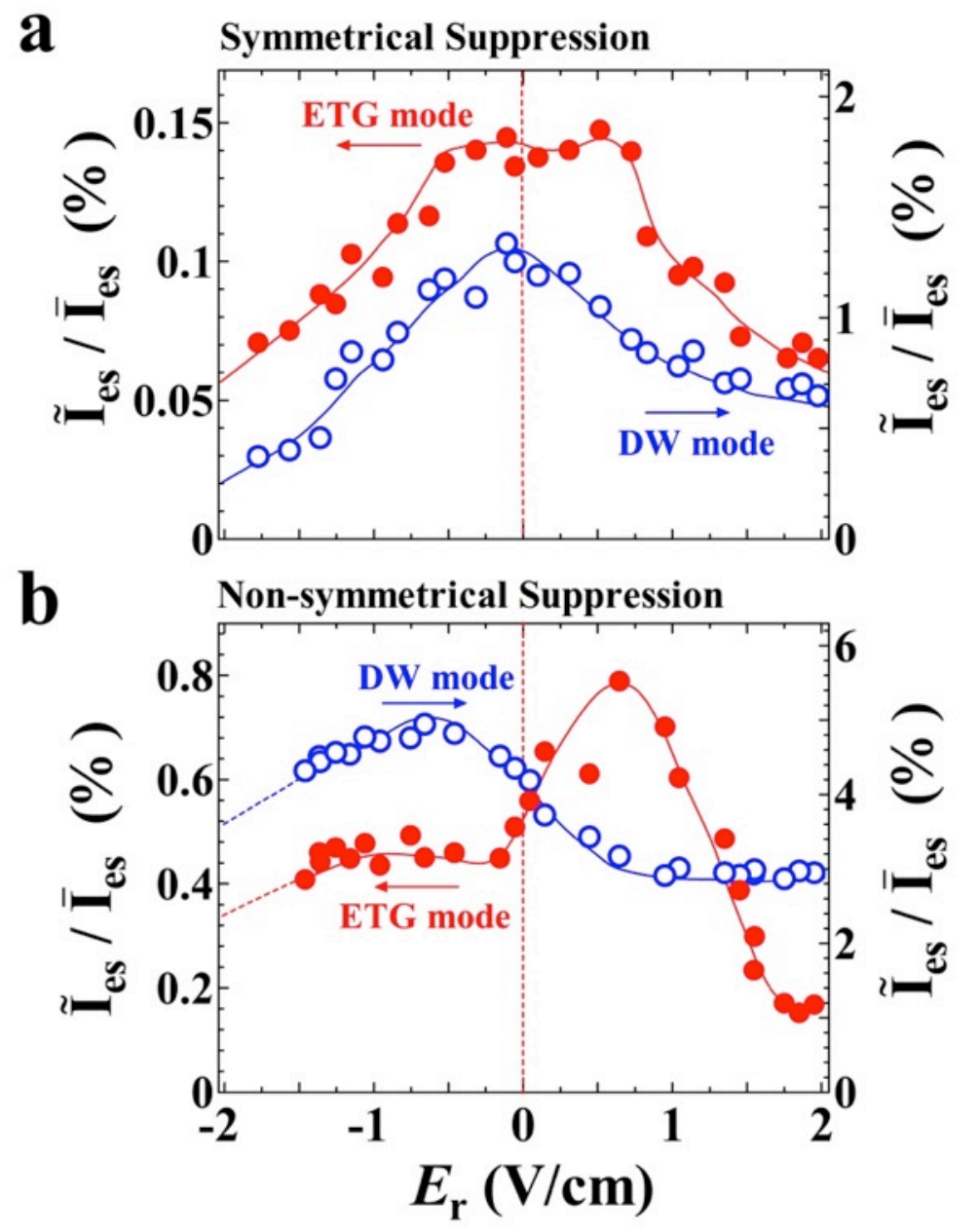

Figure 4. 


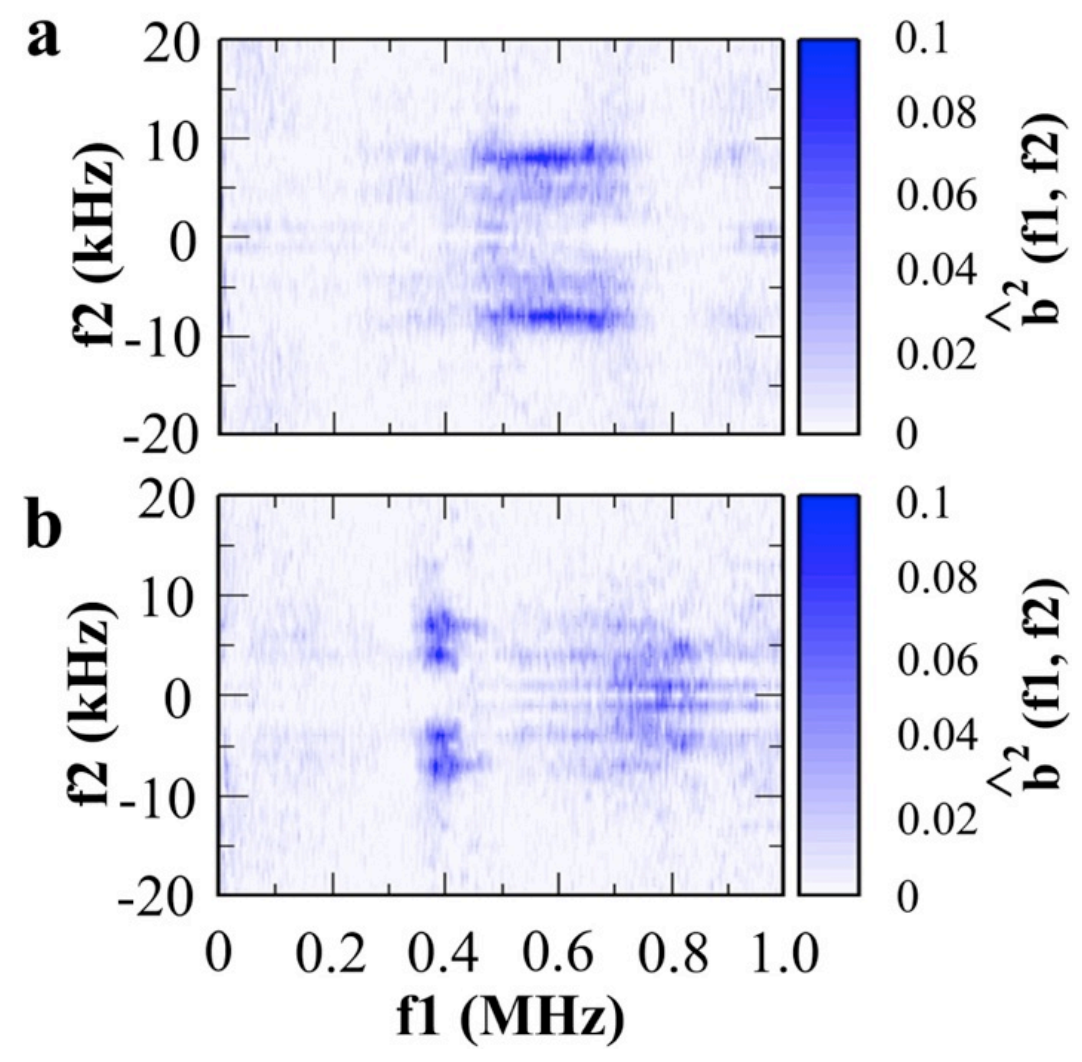

Figure 5. 

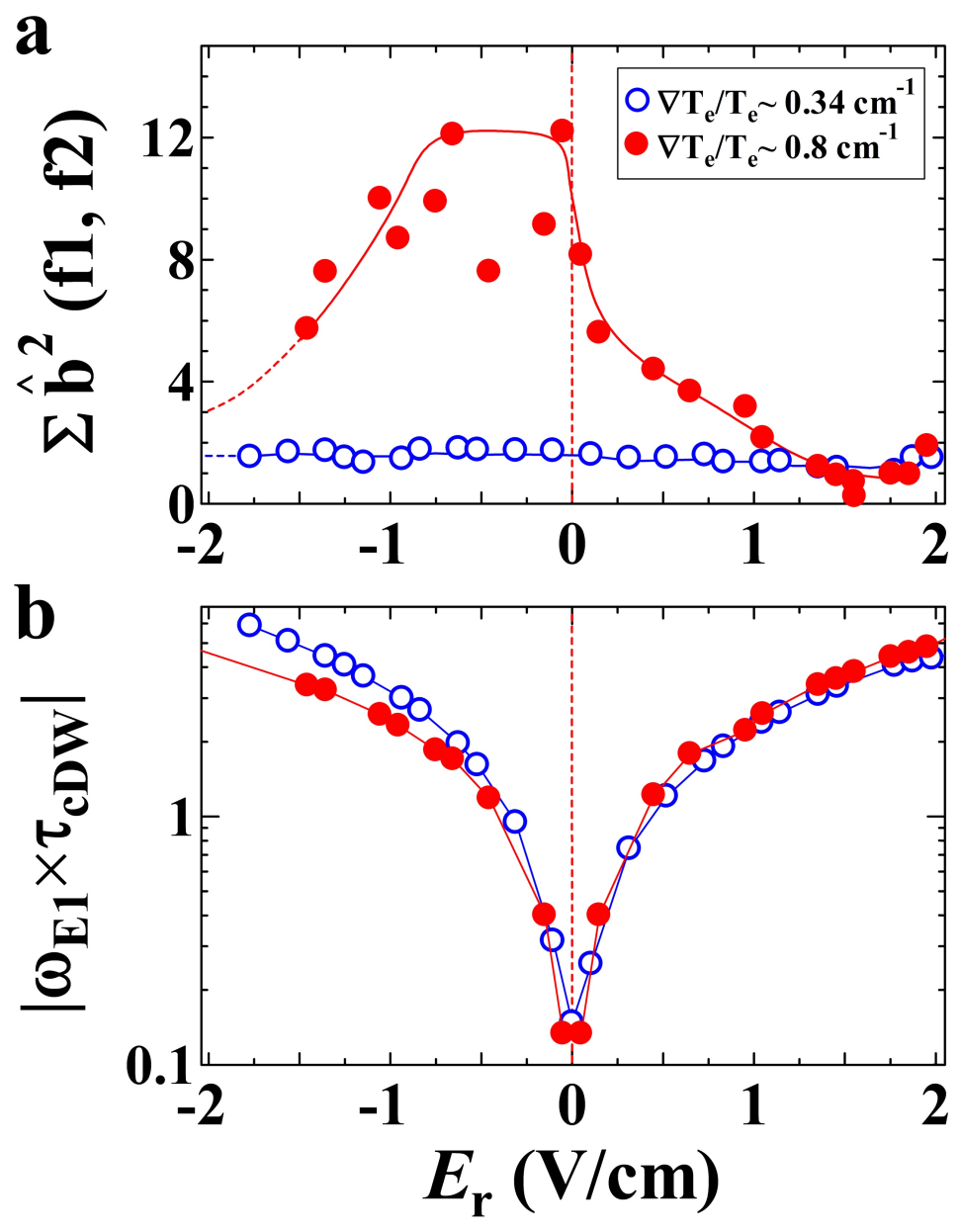

Figure 6. 

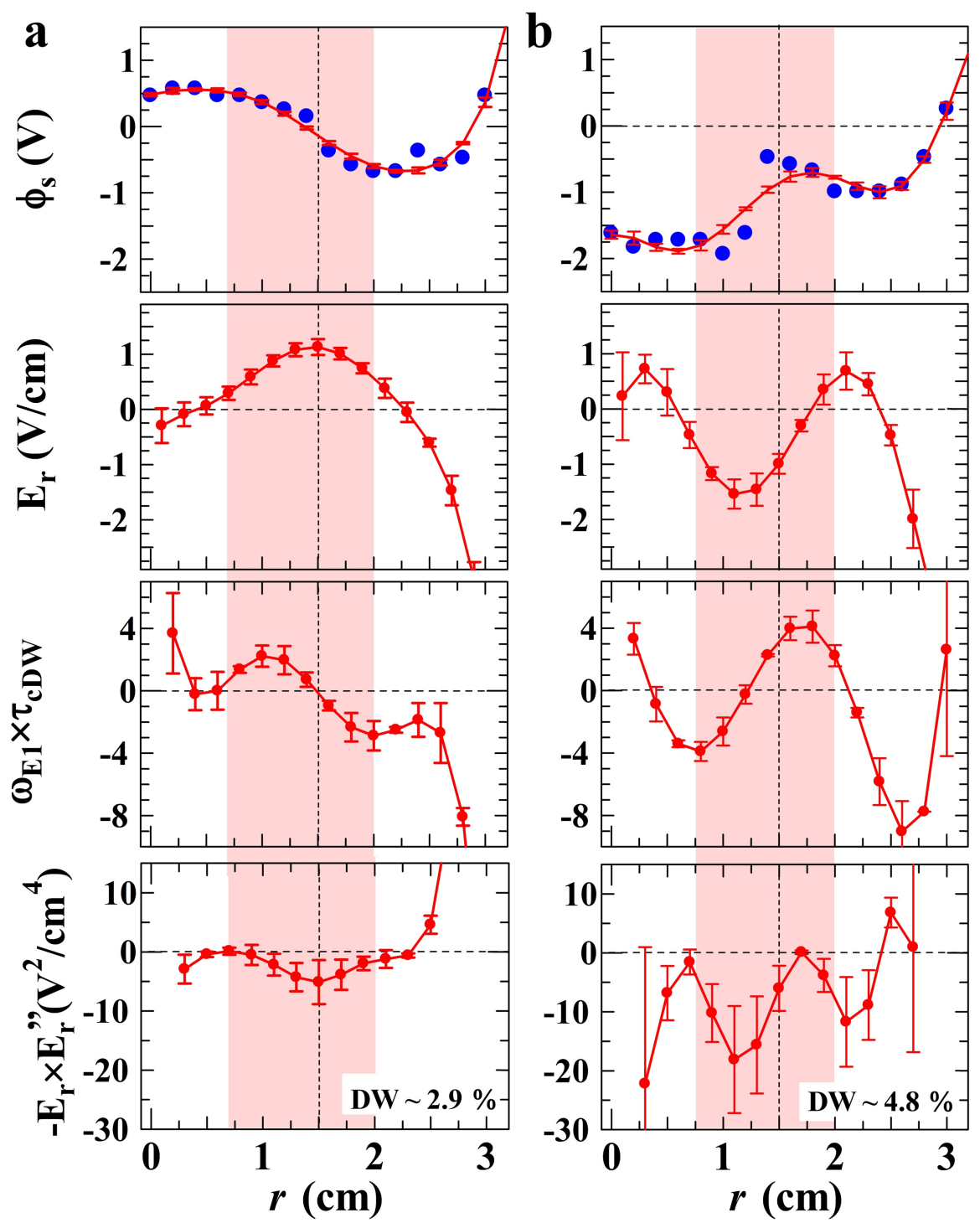

Figure 7. 